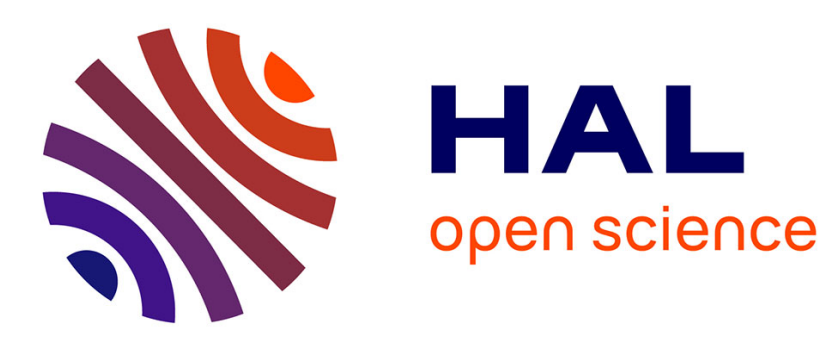

\title{
Température électronique dans une postluminescence proche de néon à une pression de quelques torrs
}

\author{
J.P. Lardy, A. Bouvier
}

\section{To cite this version:}

J.P. Lardy, A. Bouvier. Température électronique dans une postluminescence proche de néon à une pression de quelques torrs. Journal de Physique Lettres, 1976, 37 (9), pp.201-203. 10.1051/jphyslet:01976003709020100 . jpa-00231274

\section{HAL Id: jpa-00231274 https://hal.science/jpa-00231274}

Submitted on 1 Jan 1976

HAL is a multi-disciplinary open access archive for the deposit and dissemination of scientific research documents, whether they are published or not. The documents may come from teaching and research institutions in France or abroad, or from public or private research centers.
L'archive ouverte pluridisciplinaire HAL, est destinée au dépôt et à la diffusion de documents scientifiques de niveau recherche, publiés ou non, émanant des établissements d'enseignement et de recherche français ou étrangers, des laboratoires publics ou privés. 


\title{
TEMPÉRATURE ÉLECTRONIQUE DANS UNE POSTLUMINESCENCE PROCHE DE NÉON A UNE PRESSION DE QUELQUES TORRS
}

\author{
J. P. LARDY, A. BOUVIER (Mme) et A. BOUVIER \\ Laboratoire de Spectroscopie et de Luminescence $\left({ }^{*}\right)$ \\ Université Claude-Bernard, Lyon I \\ 43, bd du 11-Novembre-1918, 69621 Villeurbanne, France
}

(Reçu le 10 mai 1976, révisé le 24 mai 1976, accepté le 8 juin 1976)

\begin{abstract}
Résumé. - Un plasma de néon est créé par des ondes centimétriques, pour une gamme de pression de 0,8 à 6 torr. Des mesures d'intensité de raies n'ont pas permis de déterminer un équilibre thermodynamique local partiel; cependant en introduisant de l'hélium comme impureté dans le néon, il est possible de mesurer une température électronique. Nous obtenons $T_{\mathrm{e}}=(2350 \pm 250) \mathrm{K}$ pendant la décharge et $(2400 \pm 250) \mathrm{K}$ dans la postluminescence proche $(5 \mu \mathrm{s})$. Nous proposons une interprétation de la variation de $T_{\mathrm{e}}$ dans le temps, dans les mélanges $\mathrm{He}-\mathrm{Ne}$.
\end{abstract}

\begin{abstract}
Neon gas at pressures ranging from 0.8 to 6 torr is excited and ionized by means of microwaves pulses of duration $2 \mu \mathrm{s}$ and with a repetition rate of $800 \mathrm{~Hz}$. Partial local thermodynamic equilibrium could not be found when studying neon line intensities. But when introducing helium as an impurity it was possible to determine an electron temperature from helium line intensities. We obtained $T_{\mathrm{e}}=2350 \pm 250 \mathrm{~K}$ during the microwave discharge and $T_{\mathrm{e}}=2400 \pm 250 \mathrm{~K}$ in the early afterglow $(5 \mu \mathrm{s})$. An interpretation is proposed for the variation of $T_{\mathrm{e}}$ with time.in a Ne-He mixture.
\end{abstract}

1. Introduction. - Dans un article précédent [1] nous avons montré qu'il existait un équilibre thermodynamique local (E.T.L.) partiel dans un plasma d'hélium. Nous exposerons ici les résultats obtenus dans des plasmas créés par le même processus d'excitation dans le néon pur et dans des mélanges héliumnéon.

2. Etude du néon. - Le plasma étudié est optiquement mince pour les radiations autres que les raies de résonance, il est inhomogène et en relaxation. Nous avons tout d'abord évalué l'efficacité relative $B$ des chocs entre les atomes excités et les atomes dans l'état fondamental d'une part, et les électrons d'autre part; les calculs ont été effectués en utilisant les relations de Drawin [2] pour deux niveaux de nombre quantique principal $j$ et pour trois températures électroniques $T_{\mathrm{e}}$

\begin{tabular}{|c|c|c|}
\hline$j$ & $T_{\mathrm{e}}(\mathrm{K})$ & $B$ \\
\hline - & - & - \\
\hline \multirow[t]{3}{*}{5} & 1000 & 0,17 \\
\hline & 3000 & 0,11 \\
\hline & 10000 & 0,11 \\
\hline \multirow[t]{3}{*}{10} & 1000 & 1,30 \\
\hline & 3000 & 1,36 \\
\hline & 10000 & 1,57 \\
\hline
\end{tabular}

(*) Equipe de Recherche Associée au C.N.R.S. no 302
Les resultats du tableau ci-dessus montrent que l'influence des deux types de chocs est voisine; nous ne pourrons donc pas espérer obtenir un équilibre entre les électrons et les atomes excités dans ces niveaux.

Dans nos conditions expérimentales, la densité électronique est $n_{\mathrm{e}} \simeq 10^{12} \mathrm{~cm}^{-3}$; si les chocs électroniques étaient prépondérants l'équilibre serait atteint pour $j \geqslant 5$ à la température électronique de $2000 \mathrm{~K}$.

Si l'équilibre ne peut être atteint, ceci ne saurait provenir des phénomènes de relaxation temporelle ou spatiale. En effet :

- Le temps $\tau_{j}$ nécessaire à l'établissement de l'équilibre est faible devant la constante de déclin $\tau_{M}$ des grandeurs macroscopiques: population des métastables, température électronique $T_{\mathrm{e}}$, densité électronique $n_{\mathrm{e}}$. Pour le niveau $j=5$, nous avons en effet $\tau_{5}=2 \times 10^{-8} \mathrm{~s}$ alors que la constante de déclin des grandeurs macroscopiques est $\tau_{M} \simeq 10^{-5} \mathrm{~s}$; ainsi le temps d'établissement de l'équilibre est très faible devant le temps de variation des grandeurs macroscopiques.

- L'inhomogénéité du plasma ne perturbe pas l'équilibre si :

$$
\Lambda \geqslant\left(D \cdot \tau_{j}\right)^{1 / 2}
$$


$\Lambda=9 \times 10^{-3} \mathrm{~m}$, étant la longueur de diffusion, $D=5 \times 10^{2} \mathrm{~cm}^{3} \cdot \mathrm{s}^{-1}$ est le coefficient de diffusion mutuelle des atomes de néon.

Cette relation est bien vérifiée, car pour une température des atomes à l'état fondamental $T_{0}=1000 \mathrm{~K}$ et une pression $p=5$ torr, nous avons :

$$
\Lambda=0,9 \mathrm{~cm}, \quad \sqrt{D \cdot \tau_{j}}=1,2 \times 10^{-3} \mathrm{~cm} .
$$

La courbe de la figure 1 donnant $\log I_{j i} \lambda_{j i}^{3} / g_{j} f_{i j}$ en fonction de l'énergie des différents niveaux montre que, comme le laissait prévoir la théorie, l'équilibre n'est pas atteint pour les niveaux étudiés.

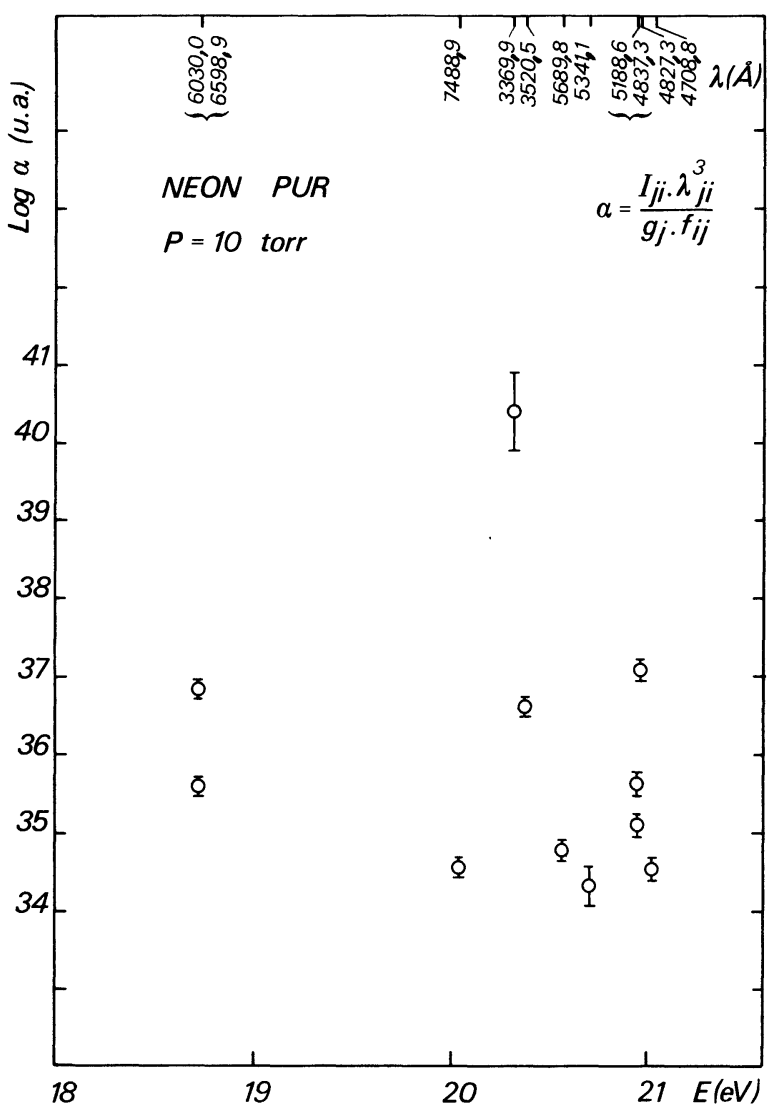

Fig. 1. - Variation expérimentale du paramètre $\log \alpha$ en fonction de l'énergie du niveau supérieur de la transition étudiée où :

$$
\alpha=I_{j i} \lambda_{j i}^{3} / g_{j} f_{i j} .
$$

$I_{j i}$ est l'intensité émise, mesurée en tenant compte de la réponse spectrale de l'appareil détecteur, $\lambda_{j i}$ la longueur d'onde de la radiation étudiée, $g_{j}$ le poids statistique de l'état supérieur, $f_{i j}$ la force d'oscillateur [3].

L'imprécision des mesures provient essentiellement de l'incertitude sur les valeurs des $f_{i j}$ et c'est l'absence de données pour les $f_{i j}$ des hauts niveaux qui a limité nos mesures.

Nous pensons donc que si un E.T.L. existe entre les électrons et les atomes de néon excités, il ne peut se produire que pour les atomes excités dans des états de nombre quantique principal supérieur à 10 , contrairement à l'hélium où l'équilibre était atteint dès $j=5$.

3. Etude des mélanges hélium-néon. - Comme nous ne pouvions pas déduire des mesures précédentes la température électronique dans un plasma de néon, nous avons introduit de l'hélium dans celui-ci à diverses concentrations. Nous constatons sur la figure 2 que, comme dans l'hélium pur, un équilibre est atteint entre les atomes dans les niveaux $j \geqslant 5$ et les électrons. La courbe de la figure 3 montre que l'hélium perturbe peu le plasma de néon, surtout en décharge où la température reste voisine de (2 $350 \pm 250) \mathrm{K}$ quelle que soit la concentration d'hélium; le potentiel d'ionisation du néon est plus faible que celui de l'hélium, c'est donc le néon qui joue le rôle principal en décharge, ce qui explique sans doute la faible perturbation provoquée par la présence d'hélium.

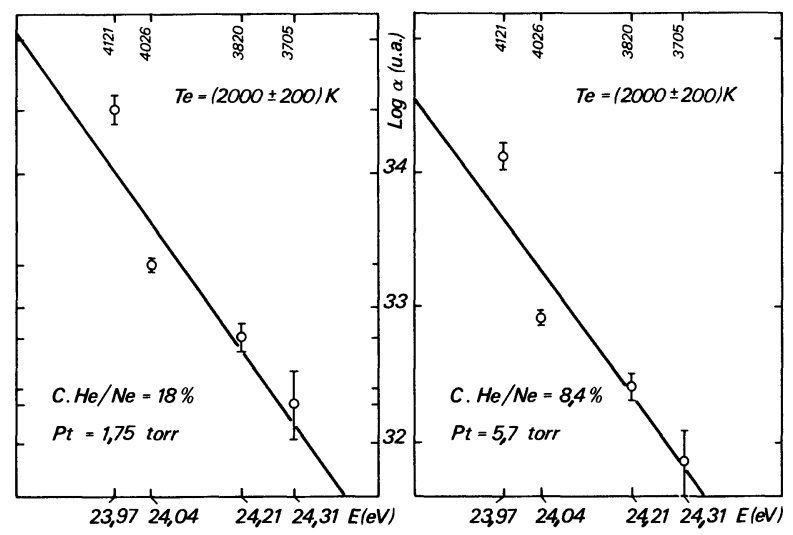

Fig. 2. - Variation expérimentale du paramètre $\log \alpha$ en fonction de l'énergie du niveau supérieur pour deux concentrations d'hélium $C=18 \%, C=8,4 \%$, pendant la décharge.

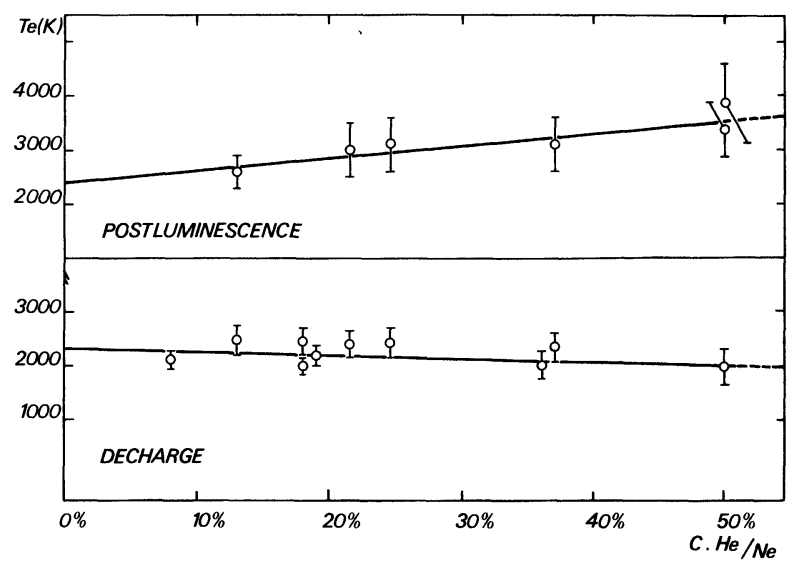

Fig. 3. - Variation de la température électronique en fonction de la concentration d'hélium pendant la décharge et pendant la postluminescence proche, $5 \mu$ s après la fin de l'impulsion.

En postluminescence ( $5 \mu$ s après la décharge), la température du plasma augmente avec la concentration d'hélium, et pour $50 \%$ d'hélium, la tempé- 
rature atteinte est celle obtenue par C. Samba [1] dans l'hélium pur. Il semble qu'au début de la postdécharge, c'est l'hélium, dès que sa concentration est de l'ordre de $20 \%$, qui commande la loi de variation de l'énergie des électrons. En effet, le gaz d'électrons se refroidit beaucoup plus vite dans l'hélium que dans le néon [4], ce que confirme le fait que le maximum d'atomes de gaz rares excités formés par recombinaison se produit beaucoup plus tôt dans l'hélium que dans le néon $[1,5]$. On en conclut que dans les mélanges $\mathrm{He}-\mathrm{Ne}$ la recombinaison est plus rapide que dans le néon pur.

Monchicourt, Touzeau et Wells [6] ont montré que dans une postluminescence d'hélium, il existe deux classes d'électrons, des électrons dits maxwelliens de faible énergie et des électrons très énergétiques créés au cours de la postluminescence. On peut penser que dans le cas du néon, il doit en être de même. La majeure partie des électrons a une énergie moyenne de quelques dizièmes d'électrons-volts; l'autre fraction, dont l'énergie est égale à quelques dizaines d'électronsvolts, est éliminée préférentiellement par diffusion et chocs sur les parois puisque la pression est basse. La recombinaison électron-ion atomique ou moléculaire est maximale pour les électrons les plus lents de la première classe qui disparaissent ainsi, ce qui peut expliquer que la température moyenne des électrons de faible énergie ne décroisse pas pendant le premier stade de la postluminescence. C'est cette température que nous mesurons; et nous trouvons, en fait, en extrapolant à concentration d'hélium nulle,

$$
T_{\mathrm{e}}=(2400 \pm 250) \mathrm{K}
$$

dans la proche postdécharge.

4. Conclusion. - En conclusion, l'introduction d'hélium sous faible concentration permet d'obtenir une température électronique moyenne, celle de la classe d'électrons donnant lieu à la recombinaison. Mais l'existence d'ions atomiques et moléculaires conduisant à la création d'espèces excitées différentes, cette température dépend certainement des diverses réactions d'équilibre.

\section{Bibliographie}

[1] Samba, C., Bouvier, A. et Bouvier, A., J. Physique 37 (1976) 359.

[2] Drawin, H. W., a) Z. Phys. 228 (1969) 99; Z. Phys. 225 (1972) 483.

Drawin, H. W. and Emard, F., b) Z. Phys. 243 (1971) 326; Z. Phys. 253 (1972) 100.
[3] Miles, B. M. and WIESE, W. L., Bibliography on Atomic Transition Probabilities, NBS Special Publ. 320 (1970).

[4] Allab, D., J. Physique 29 (1968) 22.

[5] Bouvier, A., Bouvier, A., Janin, J., C. R. Hebd. Séan. Acad. Sci. 270 (1968) 1571.

[6] Monchicourt, P., Touzeau, M. et Wells, J. Physique Colloq. 34 (1973) C2-145. 